\title{
Belphégor
}

\section{Buard, Jean-Luc. Culture médiatique et presse numérisée. Médiasphère des feuilletons-nouvelles de Marie Aycard (1794-1859)}

Vittorio Frigerio

\section{(2) OpenEdition}

Journals

Édition électronique

URL : https://journals.openedition.org/belphegor/4310

DOI : $10.4000 /$ belphegor.4310

ISSN : 1499-7185

Éditeur

LPCM

Référence électronique

Vittorio Frigerio, «Buard, Jean-Luc. Culture médiatique et presse numérisée. Médiasphère des feuilletons-nouvelles de Marie Aycard (1794-1859) », Belphégor [En ligne], 19-2 | 2021, mis en ligne le 20 décembre 2021, consulté le 20 septembre 2022. URL : http://journals.openedition.org/belphegor/ 4310 ; DOI : https://doi.org/10.4000/belphegor.4310

Ce document a été généré automatiquement le 20 septembre 2022.

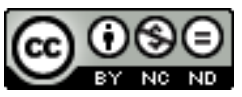

Creative Commons - Attribution - Pas d'Utilisation Commerciale - Pas de Modification 4.0 International - CC BY-NC-ND 4.0

https://creativecommons.org/licenses/by-nc-nd/4.0/ 


\title{
Buard, Jean-Luc. Culture médiatique et presse numérisée. Médiasphère des feuilletons-nouvelles de Marie Aycard (1794-1859)
}

\author{
Vittorio Frigerio
}

\section{RÉFÉRENCE}

Buard, Jean-Luc. Culture médiatique et presse numérisée. Médiasphère des feuilletonsnouvelles de Marie Aycard (1794-1859). Paris : Presses de l'Enssib, 2019. 231 p. ISBN :

978-2-37546-112-9.

1 De quoi parle-t-on quand on parle de littérature ? C'est la question qu'on pourrait se poser, en paraphrasant de manière guère originale (cela a été fait dix mille fois) le titre du célèbre recueil de nouvelles de Raymond Carver pour parler de ce livre de Jean-Luc Buard, rédacteur en chef de la revue Le Rocambole et explorateur passionné des basfonds de la production populaire. Dans ce cas particulier, la paraphrase usée peut toutefois paraître particulièrement appropriée, justement en raison de son omniprésence. C'est en effet l'histoire d'un auteur qui fut un temps omniprésent - et qui a depuis complètement disparu de la mémoire historique - que retrace ce livre, tout en proposant un discours on ne peut plus actuel sur les frontières du champ littéraire, l'idée qu'on s'en fait traditionnellement et l'influence des nouvelles méthodes de recherche sur sa redéfinition.

2 Cette étude, qui s'apparente en quelque sorte à une enquête, a pour objet, ou pour prétexte, un écrivain d'origine marseillaise nanti d'un nom inhabituel, Marie Aycard, qui lui a valu d'être cru femme par les critiques bien peu nombreux à qui il est arrivé de mentionner son nom. Buard ne lui redonne pas seulement son sexe - certifié par le collier de barbe qu'on lui voit sur une caricature signée Nadar reproduite dans le 
volume - mais s'efforce de lui restituer sa place dans le cadre de la «médiasphère " au sein de laquelle il opérait. Tâche qui le conduit, et c'est là ce qui intéresse et compte le plus dans son analyse, à reconstruire le portrait-robot de celle-ci. Aycard, dont les écrits n'ont jamais été recueillis en volume de son vivant, a été l'auteur de quelques romans feuilletons, mais surtout de « 510 chroniques et nouvelles » qui ont donné lieu à " plus de 2700 reproductions, soit quelque 3250 références cumulées dans près de 1000 journaux reproducteurs dans le monde en 17 langues dans 40 pays, de 1837 à 1960 » (153). Il apparaît comme représentatif d'une production moyenne, dans le sens de médiane, mais pas nécessairement médiocre (ce que certains critiques anglophones ont voulu appeler " middlebrow»), et surtout comme un producteur significatif d'un genre sous-estimé, le feuilleton-nouvelle, genre dont cette étude, à travers les aléas de la diffusion de l'œuvre de ce praticien, tente de restituer la position, guère secondaire, dans le cadre de la nouvelle culture médiatique de la monarchie de Juillet.

3 Jean-Luc Buard présente son propos comme celui « de positionner (ou de repositionner) le débat [entourant la définition du champ littéraire] en observant la réalité du feuilleton des années 1836-1840, une réalité "alternative", où le feuilleton ne serait pas composé de romans, mais de nouvelles » (15-16). Il se situe dans la lignée de chercheurs illustres qui l'ont précédé, tel Jean-Claude Vareille, mais surtout René Guise, et fait débuter sa démonstration par une réactualisation tout à fait bienvenue des thèses de Robert Escarpit, le sociologue de la littérature, qui trouvait tout de même quelque peu problématique que le domaine littéraire, tel que transmis par l'école et construit par la critique, ne comprenne qu'environ $1 \%$ de ce qu'une période a pu produire, et que le $99 \%$ restant passe automatiquement à la trappe. Buard - qui a, on s'en doute et le contraire serait étonnant, une petite prédilection pour le nouvelliste méconnu qu'il déterre - ne cherche toutefois guère à renverser les valeurs acquises, tâche dont l'inutilité, en plus de l'impossibilité, est avérée. Mais il souligne à raison que le type d'écriture pratiqué par Aycard, authentique best-seller dont la circulation mondiale est proprement ahurissante, peut incarner le mieux l'air du temps d'une époque et aider à se rendre compte de l'existence, déjà dans les années 30 du dixneuvième siècle, d'une sphère ou d'un univers médiatiques entièrement constitués, d'un paysage communicationnel qui réunit les pays européens et les Amériques et sert et ressert, dans un carrousel très rapide de passages, les mêmes textes au public, friand de lectures divertissantes, d'un monde qui apparaît en grande partie déjà culturellement globalisé.

4 L'exploration systématique que Jean-Luc Buard opère au sein de « ce maquis qu'est la presse populaire de la monarchie de Juillet » (51) le conduit à mettre l'accent sur un moment généralement sous-estimé de la construction de la première culture de masse. Si on savait déjà l'importance que pouvaient avoir eu les formes courtes dans le développement du roman historique, avec la publication de scènes et vignettes historiques dans les revues littéraires, telle notamment la Revue de Paris, l'importance du rôle joué par la publication de nouvelles dans les quotidiens et les hebdomadaires acquiert grâce à une recherche telle que celle-ci une dimension insoupçonnée. Pour emprunter les mots de l'auteur: "Cette guerre du format et des "standards" (feuilleton court contre feuilleton long, fiction brève contre roman de longue haleine) est l'un des aspects majeurs occultés de l'histoire de la presse » (54).

5 L'envergure de cette présence de «feuilletons-nouvelles » jusqu'ici largement ignorés ressort - et c'est là le point fort et l'originalité de ce travail - grâce à une exploration 
systématique de corpus massifs jusqu'ici impossibles d'accès ou très difficiles à consulter, nouvellement rendus disponibles par la numérisation croissante de bibliothèques et de collections entières partout de par le monde, sous l'impulsion de géants privés de la technologie comme Google ou d'acteurs nationaux comme la Bibliothèque Nationale de France à travers son site Gallica. Le flot de données qui s'offrent maintenant aux chercheurs de bonne volonté, presque effrayant par sa quantité, peut bien sûr permettre, en faisant littéralement revivre quantité de débats et de situations historiques dans leur immédiateté brute, des découvertes fascinantes. Il peut aussi, cela va de soi, mener le chercheur à se perdre dans une masse de documents éphémères à l'utilité relative, néantisant le travail de distinction, de sélection, mené par le temps. Travail discutable, toujours susceptible de remises en question et de reformulations, bien sûr, mais qui se retrouve dans ce nouveau contexte confronté au danger d'une forme de révisionnisme permanent aux conséquences en partie imprévisibles. Buard est tout à fait conscient de cela, soulignant comment ce « domaine immense, démesuré ", exige " des explorations assidues et harassantes dans des corpus d'une envergure inédite » (48) - travail qu'il appelle, en le minimisant presque au-delà du permissible, « quelque peu fastidieux » (127) ; mais il souligne fortement, et à raison, les grands avantages de ces corpus pour la recherche historique et littéraire. Cela, il va sans dire, si on sait comment les aborder pour ne pas se perdre dans leurs méandres et pour arriver à identifier à travers la masse des documents, en utilisant les bons filtres, des informations permettant des découvertes utiles.

Le personnage oublié de Marie Aycard permet, dans ce cas de figure particulier, de reconstruire tout un système de circulation des textes littéraires à l'échelle globale, éclairant le mode de fonctionnement de la presse à une époque où les droits des auteurs étaient loin d'être assurés, mais révélant aussi les particularités de la circulation de textes populaires (au sens de massivement recherchés et lus), souvent reproposés sans nom d'auteur ou sous des noms différents, traduits et retraduits, adaptés et adoptés pour et par un lectorat friand de ce genre particulier que l'histoire littéraire devait par la suite largement ignorer. Combien de fois a-t-on lu et entendu qu'à de rares exceptions près (l'inusable Maupassant, un peu de Mérimée, très peu de Balzac...) la nouvelle n'est pas un genre littéraire important dans la tradition française, au contraire de ce qui a pu se passer par exemple en Amérique du Nord, où elle a joué un rôle déterminant? En révélant, grâce à des recherches aussi étendues que paradoxalement pointues, l'importance du feuilleton-nouvelle comme précurseur $d u$ feuilleton-roman, ainsi que l'étendue surprenante de sa circulation, Buard aide à créer une image plus complète de l'évolution des formes littéraires au XIXe siècle. Cette nouvelle image conduit à se poser des questions. Comment se fait-il que ce genre, pourtant si diffusé dans la presse, ait été non seulement remplacé par le romanfeuilleton, mais totalement marginalisé et pratiquement oublié par la suite? Le discrédit relatif de la nouvelle dans la tradition littéraire française proviendrait-il éventuellement de son association avec les médias qui en étaient porteurs et avec le genre de littérature «moyenne " qui y dominait, illustré par des auteurs tels Marie Aycard? Les explorations de Buard dans cette direction rappellent les travaux menés dans le domaine italien par Claudio Gallo e Fabrizio Foni dans leur Ottocento nero italiano $^{1}$, qui avaient montré la présence forte d'une veine fantastique dans la littérature italienne - jugée pratiquement inexistante par la critique littéraire, sauf en ce qui concerne quelques rares auteurs tels Buzzati ou Landolfi - portée exclusivement par la presse, et en particulier les revues populaires à grande diffusion. Mais une 
différence importante sépare ces deux recherches, menées à une dizaine d'années de distance, en dépit de leurs conclusions voisines: la disponibilité toute récente des grandes collections de presse ancienne numérisée qui ont permis à Buard, non sans de grands efforts, d'asseoir ses hypothèses sur un socle solide fait d'une documentation à laquelle on ne pouvait, il y a encore très peu de temps, avoir accès.

7 C'est donc aussi l'aspect pratique de la recherche menée qui est illustré dans cet ouvrage, et non seulement ses résultats. L'auteur n'est pas avare de conseils, indiquant " les ressources qui ont été utiles et la manière d'y accéder et de s'en servir », tout en se posant «quelques questions épistémologiques, voire ontologiques, sur l'activité du chercheur qui utilise ces outils nouveaux» (75) - questions bienvenues. Buard estime en effet justement qu'il "convient d'élaborer des instruments de travail utiles, des guides, des méthodes de recherche innovantes, d'identifier des objets d'étude neufs » (174). Et surtout, il met en lumière une conséquence de la numérisation massive des archives de presse avec laquelle la recherche littéraire devra se confronter et qu'elle devra apprendre à gérer et à utiliser à bon escient : «La mise à disposition numérique de bibliothèques entières a un autre effet inédit : elle nivelle d'une manière radicale la globalité de leurs contenus. Elle dissout toutes les hiérarchies qualitatives. Elle met à portée d'interrogation tous les types de documents imprimés, sans égard pour leur source, leur autorité, leur légitimité, leur diffusion ou leur niveau. Ce nivellement radical est un bouleversement qui s'apparente à un cataclysme silencieux " (49). Le terme est fort, et il se trouvera des chercheurs confiants dans la stabilité pérenne des hiérarchies actuelles qui le jugeront excessif. Il nous semble toutefois que les recherches littéraires ne pourront être que bien servies par l'utilisation judicieuse et systématique de ces ressources et des archives énormes qui redeviennent chaque jour plus facilement disponibles sur la toile. L'étude de la littérature populaire pourra y trouver un souffle nouveau, et au-delà de ce domaine particulier, les recherches littéraires en général pourront à la fois élargir et approfondir leur champ d'action ${ }^{2}$. Dans cet ouvrage (qui est, il faut le rappeler, la version largement abrégée d'une thèse de doctorat en sciences de l'information et de la communication soutenue à la Sorbonne en 2015), Jean-Luc Buard parvient à montrer de manière probante ce que l'utilisation de sources numérisées peut réserver comme surprises d'envergure à qui s'y essaie sans idées préconçues. Le cas de Marie Aycard, analysé sous toutes les coutures, apparaît ainsi potentiellement généralisable, et l'auteur est entièrement justifié de conclure : «On doit poser que cet auteur-producteur n'est pas médiatiquement mineur ni secondaire. S'il a été considéré comme tel, c'est que nos instruments de lecture classiques étaient mal réglés pour apprécier son cas singulier et l'étendue de son intérêt»(185)3. Cet ouvrage entier, original et bien argumenté, est un appel, convaincant à procéder à un nouveau réglage de ces instruments, susceptible de nous aider à porter à un regard neuf sur la littérature des débuts de l'ère médiatique. 


\section{NOTES}

1. Ottocento nero italiano. Narrativa fantastica e crudele. A cura di Claudio Gallo e Fabrizio Foni. Introduzione di Luca Crovi. Milano: Nino Aragno Editore, 2009. Voir notre recension de cet ouvrage dans Belphégor Vol. 10 No. 1.

2. Un exemple récent de travail de recherche qui aurait été impensable sans l'apport déterminant des dossiers de presse numérisés est l'excellent ouvrage de Stephen Steele et Anne-Françoise Bourreau-Steele, Louis de Gonzague Frick dans tous ses états. Poète, soldat, courriériste, ami (Paris : Garnier, 2017).

3. On doit également signaler que Jean-Luc Buard et en train de procéder à une republication des feuilletons-nouvelles d'Aycard aux éditions Jacques olliveau / Mi Li Re Mi (distribution Lulu.com). Un volume, La Lettre de grâce et autres feuilletons-nouvelles du Constitutionnel (1843-1844) est déjà disponible.

\section{INDEX}

Mots-clés : Culture médiatique, presse numérisée, feuilleton, feuilleton-nouvelle, médiasphère, middlebrow

\section{AUTEUR}

\section{VITTORIO FRIGERIO}

\section{Dalhousie University}

